A generalized two-fluid picture of non-driven collisionless reconnection and its relation to whistler waves

Young Dae Yoon and Paul M. Bellan

Citation: Physics of Plasmas 24, 052114 (2017); doi: 10.1063/1.4982812

View online: http://dx.doi.org/10.1063/1.4982812

View Table of Contents: http://aip.scitation.org/toc/php/24/5

Published by the American Institute of Physics

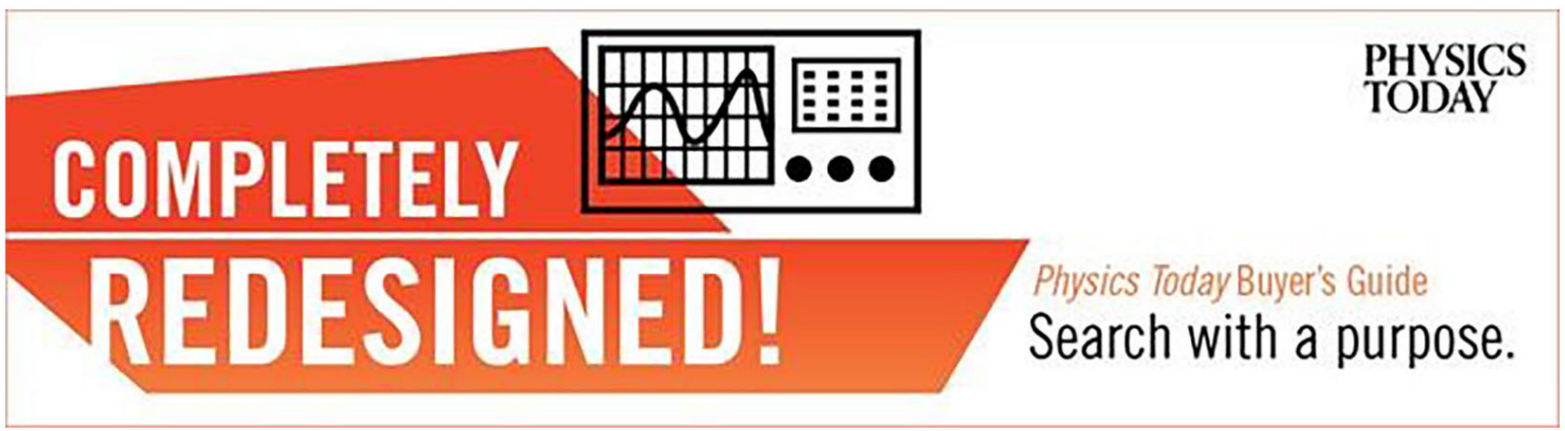




\title{
A generalized two-fluid picture of non-driven collisionless reconnection and its relation to whistler waves
}

\author{
Young Dae Yoon and Paul M. Bellan \\ Applied Physics and Materials Science, Caltech, Pasadena, California 91125, USA
}

(Received 17 February 2017; accepted 19 April 2017; published online 5 May 2017)

\begin{abstract}
A generalized, intuitive two-fluid picture of 2D non-driven collisionless magnetic reconnection is described using results from a full-3D numerical simulation. The relevant two-fluid equations simplify to the condition that the flux associated with canonical circulation $\mathbf{Q}=m_{e} \nabla \times \mathbf{u}_{e}+q_{e} \mathbf{B}$ is perfectly frozen into the electron fluid. In the reconnection geometry, flux tubes defined by $\mathbf{Q}$ are convected with the central electron current, effectively stretching the tubes and increasing the magnitude of $\mathbf{Q}$ exponentially. This, coupled with the fact that $\mathbf{Q}$ is a sum of two quantities, explains how the magnetic fields in the reconnection region reconnect and give rise to strong electron acceleration. The $\mathbf{Q}$ motion provides an interpretation for other phenomena as well, such as spiked central electron current filaments. The simulated reconnection rate was found to agree with a previous analytical calculation having the same geometry. Energy analysis shows that the magnetic energy is converted and propagated mainly in the form of the Poynting flux, and helicity analysis shows that the canonical helicity $\int \mathbf{P} \cdot \mathbf{Q} d V$ as a whole must be considered when analyzing reconnection. A mechanism for whistler wave generation and propagation is also described, with comparisons to recent spacecraft observations. Published by AIP Publishing. [http://dx.doi.org/10.1063/1.4982812]
\end{abstract}

\section{INTRODUCTION}

Magnetic reconnection is a plasma phenomenon in which magnetic field lines break and reconnect at a critical location, releasing large amounts of magnetic energy. This process is ubiquitous in both naturally occurring plasmas and laboratory plasmas; important examples are the solar corona, ${ }^{1,2}$ the Earth's magnetosphere, ${ }^{3}$ tokamaks, ${ }^{4}$ reversed field pinches, ${ }^{5}$ spheromaks, ${ }^{6,7}$ and field reversed configurations. ${ }^{8}$

According to ideal MHD, magnetic flux is frozen into the plasma which means that plasma cannot move across magnetic field lines and equivalently, magnetic field lines cannot move across plasma. Magnetic reconnection involves violation of this frozen-in condition, and because electrical resistivity, a mechanism based on electron-ion collisions, allows magnetic field lines to diffuse across plasma, it had long been regarded as the basic enabling mechanism for magnetic reconnection. ${ }^{9}$ However, observed reconnection rates have been observed to be much faster than those predicted by this resistive diffusion model, ${ }^{4,9}$ and the discrepancy led to a search for much faster mechanisms. It is now generally agreed that non-MHD, collisionless effects such as the Hall term and finite electron inertia are responsible for fast reconnection. These effects become important at small length scales such as the ion Larmor radius or the ion skin depth where MHD is no longer valid. For example, in a recent set of experiments involving a plasma jet, ${ }^{7,10}$ a kinkinduced Rayleigh-Taylor instability ${ }^{11}$ effectively narrowed the plasma column to the ion skin depth scale, transitioning from an MHD-governed system to a system governed by Hall and electron inertia effects. A strong suggestion that Hall and electron inertia terms were important was the observed emission of circularly polarized whistler waves in association with the reconnection. The importance of the microscopic scale was evident in this experiment, since reconnection only occurred when the plasma current channel width was reduced to be of the order of the ion skin depth.

Canonical circulation $\mathbf{Q}=m_{\sigma} \nabla \times \mathbf{u}_{\sigma}+q_{\sigma} \mathbf{B}^{12,13}$ — which is the curl of the canonical momentum $\mathbf{P}=m_{\sigma} \mathbf{u}_{\sigma}+q_{\sigma} \mathbf{A}-$ has long been known to be an important quantity in two-fluid models of magnetic helicity and collisionless magnetic reconnection. ${ }^{14-19}$ In particular, $\mathbf{Q}$ was found to convect with the electron fluid in the EMHD regime, ${ }^{20}$ with the important repercussion that localized violation of the topological constraints of $B$ can occur without a dissipative mechanism. The present paper provides an important addition to previous studies by focussing attention on the temporal evolution of the flux tubes defined by $\mathbf{Q}$. It will explicitly be shown via numerical/analytical calculation and 3D graphics that the coupling of these flux tubes to the central electron current generates the quadrupole magnetic fields ${ }^{9}$ associated with magnetic reconnection and furthermore increases $\mathbf{Q}$ exponentially by stretching and lengthening the flux tubes. Also, the two-constituent nature of $\mathbf{Q}$ will explicitly be used to explain various aspects of collisionless reconnection.

Whistler waves have often been proposed to be associated with two-fluid magnetic reconnection, but the details of this association have been unclear and controversial. For example, by analyzing results from a two-dimensional particle-in-cell simulation, Drake et al. $^{21}$ argued that whistler waves mediate reconnection, relating the outflow region of the reconnection $\mathrm{x}$-point to standing whistler half-periods. However, Fujimoto and Sydora ${ }^{22}$ in a similar simulation observed whistler waves in the downstream region of the flow, and Bellan ${ }^{23}$ showed analytically that 2D Hall reconnection is a purely growing instability rather than a wave.

In this paper, a generalized two-fluid picture for $2 \mathrm{D}$ nondriven collisionless magnetic reconnection is presented with 
particular attention to the temporal evolution of the flux tubes defined by $\mathbf{Q}$ and the interaction between the two terms constituting canonical circulation. Numerically calculated reconnection rates are compared with a previous analytical work $^{23}$ having the same geometry. Energy and helicity calculations are also presented. Whistler wave generation and propagation is observed and explained.

This paper is organized as follows: Section II rearranges a set of two-fluid equations to obtain a single dimensionless differential equation for the magnetic field and relates this to the frozen-in condition of the canonical circulation flux. The numerical methods used are described in detail, along with the validation of the computer code in a fully 3D, linear regime. Section III A first shows the main results of the simulation in a non-linear regime and compares them to previous studies. Section III B then constructs a generalized, intuitive picture for 2D non-driven collisionless reconnection, focussing on $\mathbf{Q}$ flux conservation leading to exponential growth and its two-constituent nature. Relatively simple explanations for some phenomena (e.g., spiked central current filaments ${ }^{15,16,18,24}$ ) are given as well. Section III C shows that the magnetic energy is converted and propagated mainly in the form of Poynting flux, and that the canonical helicity $\int \mathbf{P} \cdot \mathbf{Q} d V$ as a whole must indeed be considered rather than just the magnetic helicity $\int \mathbf{A} \cdot \mathbf{B} d V .^{13,25}$ Section III D proposes a mechanism for whistler wave generation and propagation associated with reconnection and shows that it agrees with magnetosheath and magnetosphere observations. Section IV summarizes the results and discusses some prospects on 3D-localized reconnection in a similar regime.

\section{MODEL AND VALIDATION}

\section{A. Model and assumptions}

The full-3D simulation is conducted in a 2D two-fluid regime where ions remain stationary, so electron motion provides all the necessary fields. This assumption is valid at length scales smaller than the ion skin depth, and it is in this regime that the Hall term and the electron inertia term become important. The significance of the Hall term can be seen by comparing the $\mathbf{U} \times \mathbf{B}$ term in the generalized Ohm's law to the Hall term $\mathbf{J} \times \mathbf{B} / n e$; assuming $\mathbf{U}$ is the order of the Alfvén velocity, the $\mathbf{U} \times \mathbf{B}$ term goes like $B^{2} / \sqrt{\mu_{0} m_{i} n}$, and the Hall term like $B^{2} / \mu_{0} n e L$, where $L$ is the typical length scale of magnetic field variations. After some rearrangement, it can easily be seen that the two terms become comparable when $L \approx c / \omega_{p i}=d_{i}$.

Electron inertia is also included since, as has been shown by previous simulations, ${ }^{15,18}$ the length scale of the current density layer becomes some multiple of the electron skin depth in the collisionless reconnection process. Since $\left|m_{e} \mathbf{u}_{e}\right| /\left|q_{e} \mathbf{A}\right| \sim m_{e} / \mu_{0} n q_{e}^{2} L^{2}=d_{e}^{2} / L^{2}$, a narrow current layer means that the mechanical term-which is represented by finite electron inertia-in the canonical momentum $\mathbf{P}=m_{e} \mathbf{u}_{e}$ $+q_{e} \mathbf{A}$ becomes important.

The plasma is also assumed to be incompressible. This is because compression in this two-fluid regime would mean a charge build-up, yielding electrostatic electric fields. However, in Ampère's law, $\nabla \times \mathbf{B}=\mu_{0} \mathbf{J}+\mu_{0} \varepsilon_{0}$ $\partial \mathbf{E} / \partial t$, the displacement current is much smaller than the electron current at velocities much smaller than the light speed, which is assumed to be the case. Since only electrons are moving, incompressibility corresponds to having $\nabla \cdot \mathbf{J}=0$. The effect of compressibility has been studied in Refs. 26-28, in which compressibility introduces a new skin depth parameter $\lambda_{e}>d_{e}$. It was found that this effectively reduces the reconnection rate, broadens the eigenmode structure, and stabilizes instabilities in the nonlinear stage of reconnection.

Magnetic reconnection is modelled as a perturbation to a 2D Harris current sheet. ${ }^{29}$ The background magnetic field is therefore

$$
\mathbf{B}_{0}(x)=B_{0} \tanh \left(x / L_{x}\right) \hat{y},
$$

where $L_{x}$ is the reconnection half-length scale. To ensure zero divergence of the magnetic field, the perturbation term is expressed as a vector potential in the Coulomb gauge in the $\mathrm{z}$-direction ${ }^{30}$

$$
A_{z}(x, y)=-\varepsilon B_{0} L_{x} \exp \left[-x^{2} / 2 L_{x}^{2}-y^{2} / 2 L_{y}^{2}\right],
$$

where $L_{y}$ is the half-length scale of the change in the current filament in the $y$ direction, and $\varepsilon$ is the initial relative perturbation strength.

\section{B. Equation for the magnetic field}

Based on the above assumptions, we now re-derive a single dimensionless differential equation for the perturbed magnetic field. We start with Faraday's law, Ampère's law, and the generalized Ohm's law including the Hall, electron inertia, and pressure terms

$$
\begin{gathered}
\nabla \times \mathbf{E}=-\frac{\partial \mathbf{B}}{\partial t}, \\
\nabla \times \mathbf{B}=-\mu_{0} n e \mathbf{u}, \\
\mathbf{E}+\mathbf{u} \times \mathbf{B}=-\frac{m_{e}}{e} \frac{D \mathbf{u}}{D t}-\frac{\nabla P}{n e} .
\end{gathered}
$$

Here, u represents the electron velocity, and using Eqs. (1) and (4) the electron current is initially $\mathbf{u}_{0}=-B_{0} \hat{z} /$ $\left(\mu_{0} n e L \cosh ^{2}(x / L)\right)$. Note that the Hall term is the electron Lorentz force, since current is only dependent on electron velocity in this regime. Taking the curl of Eq. (5), applying Eq. (3), and then substituting u using Eq. (4) gives

$$
-\frac{\partial \mathbf{B}}{\partial t}=\frac{m_{e}}{\mu_{0} n e^{2}} \nabla \times \frac{D(\nabla \times \mathbf{B})}{D t}+\nabla \times\left[\frac{\nabla \times \mathbf{B}}{\mu_{0} n e} \times \mathbf{B}\right] .
$$

Note that the pressure term disappeared when taking the curl. Here, we notice that $m_{e} /\left(\mu_{0} n e^{2}\right)=c^{2} / \omega_{p e}^{2}=d_{e}^{2}$, and $B_{y} /\left(\mu_{0} n e\right)=d_{e}^{2}\left|\omega_{c e}\right|$. Then, normalizing length by $d_{e}$, time by $\left|\omega_{c e}\right|^{-1}$, magnetic field by $B_{0}$, using $\bar{\nabla} \times(\bar{\nabla} \times \overline{\mathbf{B}})=-\bar{\nabla}$ $\times \overline{\mathbf{u}}=-\bar{\nabla}^{2} \overline{\mathbf{B}}$, and decomposing the convective derivative into $\partial / \partial t+\mathbf{u} \cdot \nabla$ gives 


$$
\begin{aligned}
-\frac{\partial \overline{\mathbf{B}}}{\partial \bar{t}}= & \bar{\nabla} \times \frac{\partial(\bar{\nabla} \times \overline{\mathbf{B}})}{\partial \bar{t}}+\bar{\nabla} \times[\overline{\mathbf{u}} \cdot \bar{\nabla}(\bar{\nabla} \times \overline{\mathbf{B}})] \\
& +\bar{\nabla} \times[(\bar{\nabla} \times \overline{\mathbf{B}}) \times \overline{\mathbf{B}}] \\
= & -\frac{\partial \bar{\nabla}^{2} \overline{\mathbf{B}}}{\partial \bar{t}}-\bar{\nabla} \times[\overline{\mathbf{u}} \cdot \bar{\nabla} \overline{\mathbf{u}}]+\bar{\nabla} \times[(\bar{\nabla} \times \overline{\mathbf{B}}) \times \overline{\mathbf{B}}] \\
= & -\frac{\partial \bar{\nabla}^{2} \overline{\mathbf{B}}}{\partial \bar{t}}-\bar{\nabla} \times\left[\bar{\nabla}\left(\frac{\bar{u}^{2}}{2}\right)-\overline{\mathbf{u}} \times(\nabla \times \overline{\mathbf{u}})\right] \\
& +\bar{\nabla} \times[(\bar{\nabla} \times \overline{\mathbf{B}}) \times \overline{\mathbf{B}}] \\
= & -\frac{\partial \bar{\nabla}^{2} \overline{\mathbf{B}}}{\partial \bar{t}}+\bar{\nabla} \times\left[\overline{\mathbf{u}} \times \bar{\nabla}^{2} \overline{\mathbf{B}}\right]+\bar{\nabla} \times[(\bar{\nabla} \times \overline{\mathbf{B}}) \times \overline{\mathbf{B}}],
\end{aligned}
$$

where barred quantities are dimensionless. Upon rearranging, this becomes

$$
\frac{\partial}{\partial \bar{t}}\left(\bar{\nabla}^{2} \overline{\mathbf{B}}-\overline{\mathbf{B}}\right)=-\bar{\nabla} \times\left[(\bar{\nabla} \times \overline{\mathbf{B}}) \times\left(\bar{\nabla}^{2} \overline{\mathbf{B}}-\overline{\mathbf{B}}\right)\right] .
$$

This is the dimensionless differential equation that is to be solved numerically and corresponds to Eq. (7) in Ref. 14 for a perfectly conducting regime.

Defining $\mathbf{Q}=\bar{\nabla}^{2} \overline{\mathbf{B}}-\overline{\mathbf{B}}$, Eq. (8) can be written in the familiar form

$$
\frac{\partial \mathbf{Q}}{\partial \bar{t}}=\bar{\nabla} \times(\overline{\mathbf{u}} \times \mathbf{Q}),
$$

which, by comparison to the plasma induction equation $\partial \mathbf{B} / \partial t=\nabla \times(\mathbf{U} \times \mathbf{B})$, means that the flux associated with $\mathbf{Q}$ is frozen into fluid moving with velocity $\mathbf{u}$ just like the flux associated with $\mathbf{B}$ is frozen into fluid moving with velocity $\mathbf{U}$ in ideal MHD. Since

$$
\begin{aligned}
\mathbf{Q} & =\bar{\nabla}^{2} \overline{\mathbf{B}}-\overline{\mathbf{B}} \\
& =\bar{\nabla} \times \overline{\mathbf{u}}-\overline{\mathbf{B}} \\
& =\bar{\nabla} \times \overline{\mathbf{u}}-\bar{\nabla} \times \overline{\mathbf{A}} \\
& =\bar{\nabla} \times \mathbf{P},
\end{aligned}
$$

where $\mathbf{P}$ is the normalized canonical momentum, canonical circulation flux $C=\int \mathbf{Q} \cdot d \mathbf{s}$ is frozen into the electron velocity in this two-fluid regime. Therefore, flux tubes defined by canonical circulation cannot reconnect. This property has been observed in previous studies ${ }^{15,18,19}$ where quantities related to $\mathbf{Q}$ in the $x-y$ plane were mainly considered, but here we will focus on the three-dimensional $\mathbf{Q}$ flux tube behavior and how this behavior affects various associated quantities.

The fact that the canonical circulation flux $C$ consists of two terms- the electron circulation $\int d \mathbf{s} \cdot \nabla \times \mathbf{u}$ and the magnetic flux $\int d \mathbf{s}$. B-is extremely important in analyzing magnetic reconnection. Although $C$ is conserved by Eq. (9), the two terms constituting $C$ may individually change such that one term becomes smaller while the other becomes larger so that their sum remains the same. ${ }^{19,26}$ In particular, magnetic reconnection can be seen as the conversion of magnetic flux into electron circulation while maintaining constant $C$ within a canonical momentum flux tube. What has classically been called the diffusion region will henceforth be called "the conversion region" in this paper.

Also, Eq. (9) is completely mass-independent, and, for example, it could have been for example normalized in length and time by $d_{i}$ and $\omega_{c i}^{-1}$, on multiplying the underlying equations by $m_{i} / m_{e}$ (although Eq. (4) would be different, yielding a slightly different equation).

To find $\mathbf{E}$ and $P$, we normalize the induced electric field and pressure by $\overline{\mathbf{E}}=e \mathbf{E} / m_{e} d_{e}\left|\omega_{c e}\right|^{2}$ and $\bar{P}=P / d_{e}^{2}$ $\left|\omega_{c e}\right|^{2} m_{e} n_{e}$ and simplify Eq. (5) to (dropping bars)

$$
\mathbf{E}+\mathbf{u} \times \mathbf{B}=-\frac{D \mathbf{u}}{D t}-\nabla P,
$$

where the barotropic assumption was used so that $\nabla P / n$ $=\nabla\left(\int d P / n\right)$. Taking the divergence of Eq. (11) and using $\nabla \cdot \mathbf{E}=0$ gives the following relation for the scalar pressure:

$$
\nabla^{2}\left(P+\frac{u^{2}}{2}\right)=\nabla \cdot[\mathbf{u} \times \mathbf{Q}] .
$$

Knowing $P$, we can calculate $\mathbf{E}$ using Eq. (11).

Dotting Eq. (11) with $\mathbf{u}$ and rearranging gives the following energy equation:

$$
\frac{\partial}{\partial t}\left(\frac{u^{2}}{2}+\frac{B^{2}}{2}\right)+\nabla \cdot\left(\frac{u^{2}}{2} \mathbf{u}+P \mathbf{u}+\mathbf{E} \times \mathbf{B}\right)=0 .
$$

From left to right each term represents respectively the change in the internal electron kinetic energy, the magnetic potential energy, the electron kinetic energy flux, the pressure flux, and the Poynting flux. In comparison to the MHD energy principle, ${ }^{31}$ the $\frac{\partial}{\partial t}\left(\frac{P}{\gamma-1}\right)$ term seems to be missing in this equation. This is because incompressibility dictates that $\gamma \rightarrow \infty$ in the adiabatic relation $P \sim \rho^{\gamma}$, since we need infinite pressure to change $\rho$. The pressure convection term $\nabla \cdot\left(\frac{\gamma}{\gamma-1} P \mathbf{U}\right)$ also changes to $\nabla \cdot(P \mathbf{u})$ in this limit.

The conserved helicity (canonical helicity) in this regime is ${ }^{12,13,32}$

$$
K=\int \mathbf{P} \cdot \mathbf{Q} d V=\int[\mathbf{u} \cdot \nabla \times \mathbf{u}-\mathbf{A} \cdot \nabla \times \mathbf{u}-\mathbf{u} \cdot \mathbf{B}+\mathbf{A} \cdot \mathbf{B}] d V,
$$

where the first term is the hydrodynamical helicity density, and the final two terms are the current density helicity density and the magnetic helicity density, respectively.

\section{Numerical method}

Equation (8) was solved on a Cartesian grid with typical size $(x, y, z)=(60,180,10)$ in full 3D. The spatial and temporal step sizes were respectively set to $(0.5,0.5,2) d_{e}$ and $0.2\left|\omega_{c e}\right|^{-1}$, which meet the criteria $\omega_{c e} \Delta t \ll 1$ (slower than electron cyclotron frequency) and $\omega_{c e} \Delta t<\Delta x^{2} / d_{e}^{2}$ (slower than whistler propagation). ${ }^{33}$ The fully non-linear code was written with the ultimate goal of simulating a 3D-localized 
reconnection. The present paper first discusses a fully 3D linear situation where a localized source excites a linear wave; this is compared to results of a lab experiment and used as a way of validating the code in the linear regime. The paper then considers a fully non-linear situation where there is no dependence on the $z$ coordinate so that comparisons can be made to previous work on two-fluid reconnection; this analysis produces some interesting new insights involving frozenin canonical circulation flux and excitation of whistler waves.

For a given initial magnetic field configuration, we first calculate $\mathbf{Q}=\bar{\nabla}^{2} \overline{\mathbf{B}}-\overline{\mathbf{B}}$. From this we then calculate the right-hand side of Eq. (9) and define the resultant vector as $\mathbf{R}(\mathbf{r}, t)$. Spatial derivatives were evaluated using the finite central difference scheme and temporal derivatives using the finite forward difference scheme. We then advance $\mathbf{Q}$ in time using $\partial \mathbf{Q} / \partial t=\mathbf{R}$. Using the new value of $\mathbf{Q}$ we then solve for $\overline{\mathbf{B}}$ in the next time step by iterative integration of the top line of Eq. (10) using the relaxation method. ${ }^{34}$ Neumann boundary conditions were used. The numerical error between each step in the iterative relaxation integration was found to be less than $1 \%$ across the entire grid. Improvement on this error could easily be achieved by simply running the integration for a longer time, but it was found that the percentage improvement yielded little change compared to the increase in computation time. The code was written in MATLAB R2016b and run on a desktop Pentium V computer.

\section{Dispersion relation and whistler wave test}

The validity of the code is first checked by comparing to known linear results. Setting $\overline{\mathbf{u}}_{0}=0$ and $\overline{\mathbf{B}}_{0}=\hat{z}$ after linearizing Eq. (8) and Fourier analyzing yields the following dimensionless dispersion relation:

$$
\bar{k}^{2}=\frac{1}{\frac{1}{\bar{\omega}} \cos \theta-1},
$$

where $\theta$ is the angle the $\mathbf{k}$ vector makes with respect to the background field. When restored to dimensioned quantities, i.e., $\bar{k} \rightarrow k d_{e}$ and $\bar{\omega} \rightarrow \omega /\left|\omega_{c e}\right|$ Eq. (15) becomes the whistler wave dispersion relation

$$
\frac{c^{2} k^{2}}{\omega^{2}}=\frac{\omega_{p e}^{2}}{\omega\left(\left|\omega_{c e}\right| \cos \theta-\omega\right)} .
$$

Stenzel and Urrutia $^{35}$ recently reported laboratory experiments where whistler waves were excited by small loop antennas in unbounded plasmas in a uniform background magnetic field. The numerical code was arranged to model the morphology of the antenna currents in the experiment, and the experimental wave propagation observations were successfully replicated. In particular, the code was able to produce resonance cone ${ }^{36}$ whistlers arising from finite electron inertia, as well as the Gendrin modes ${ }^{37}$ which were the main focus of the experiment.

An analytical wave solution for the experiment conditions was also derived, involving Fourier transforms in the parallel and perpendicular directions and contour integrals. The resultant integral solution for $B_{\phi}$ is given in the Appendix and this integral was numerically integrated. The solution exhibited the resonance cone with the correct cone angle. The numerical solution agreed very well with the analytical solution, except that the analytical solution was sharper since its source was a delta function whereas the source for the numerical solution was a Gaussian.

\section{RECONNECTION RESULTS}

The code was modified to simulate two-dimensional reconnection and was compared to previous predictions (e.g., Ref. 23). For most simulation runs, the grid size was set to $(x, y, z)=(30,90,20) d_{e}, L_{x}=3 d_{e}, L_{y}=10 d_{e}$, and $T_{\max }=400\left|\omega_{c e}\right|^{-1}$. The small parameter $\varepsilon$ was initially set to 0.01 so that Eq. (2) would give a slight perturbation to the Harris current sheet.

\section{A. Reconnection rate}

Figure 1 shows the time evolution of the magnetic field streamlines after the perturbation. The color represents the height ( $z$-direction) of the streamline location, yellow for positive and blue for negative. Some streamlines are cut off as they exit the grid. The field of view is zoomed in for clarity of presentation. The smaller plots on the right side of each plot are the $2 \mathrm{D}$ streamlines in the $y-z$ plane. The field lines spontaneously come together and reconnect, showing that the process is indeed an instability. The lines after reconnection convect in the electron flow direction (red arrow) giving rise to quadrupole out-ofplane fields, agreeing with previous simulations and experiments. $^{22,38,39}$

The reconnection rate was calculated by measuring the $\log$ of the time dependence of the outflow electron velocity and doing a linear fit to the middle third in time, the slope of which gives the instability rate. The reason for excluding the earlier and latter thirds is because we start the simulation with an initial perturbation without an initial rise rate, so the code makes the perturbation rise much faster in the earlier times, and in the latter third, the linear theory used in Refs. 17 and 23 is not valid as the outflow velocity becomes comparable to the initial current sheet velocity. It is worth mentioning here that strictly speaking, one has to distinguish between the "magnetic" reconnection rate-where the increase rate of reconnected flux contours are concernedand the particle escape rate in this regime, since the unmagnetized nature of the escaping particles means that there is no necessary correlation between the two. We define the reconnection rate to be the latter in this paper. This definition of rate as an e-folding time also should not be confused with the definition used for steady-state problems where the rate is the Alfvèn Mach number of the inflow. ${ }^{40}$

The time scale was simulated for different $L_{x}$ and $L_{y}$, and was compared to that of Bellan, ${ }^{23}$ where the growth rate was calculated to be $\gamma_{c} / \omega_{c e}=\frac{\sqrt{2}}{\kappa} \frac{\pi d_{e}^{2}}{2 L_{y} L_{x}}$, where $\kappa$ was solved numerically in the paper for varying parameters. This rate was found to be on the order of a whistler-like frequency. Table I shows the comparison between the calculated $\gamma_{c}$ and 

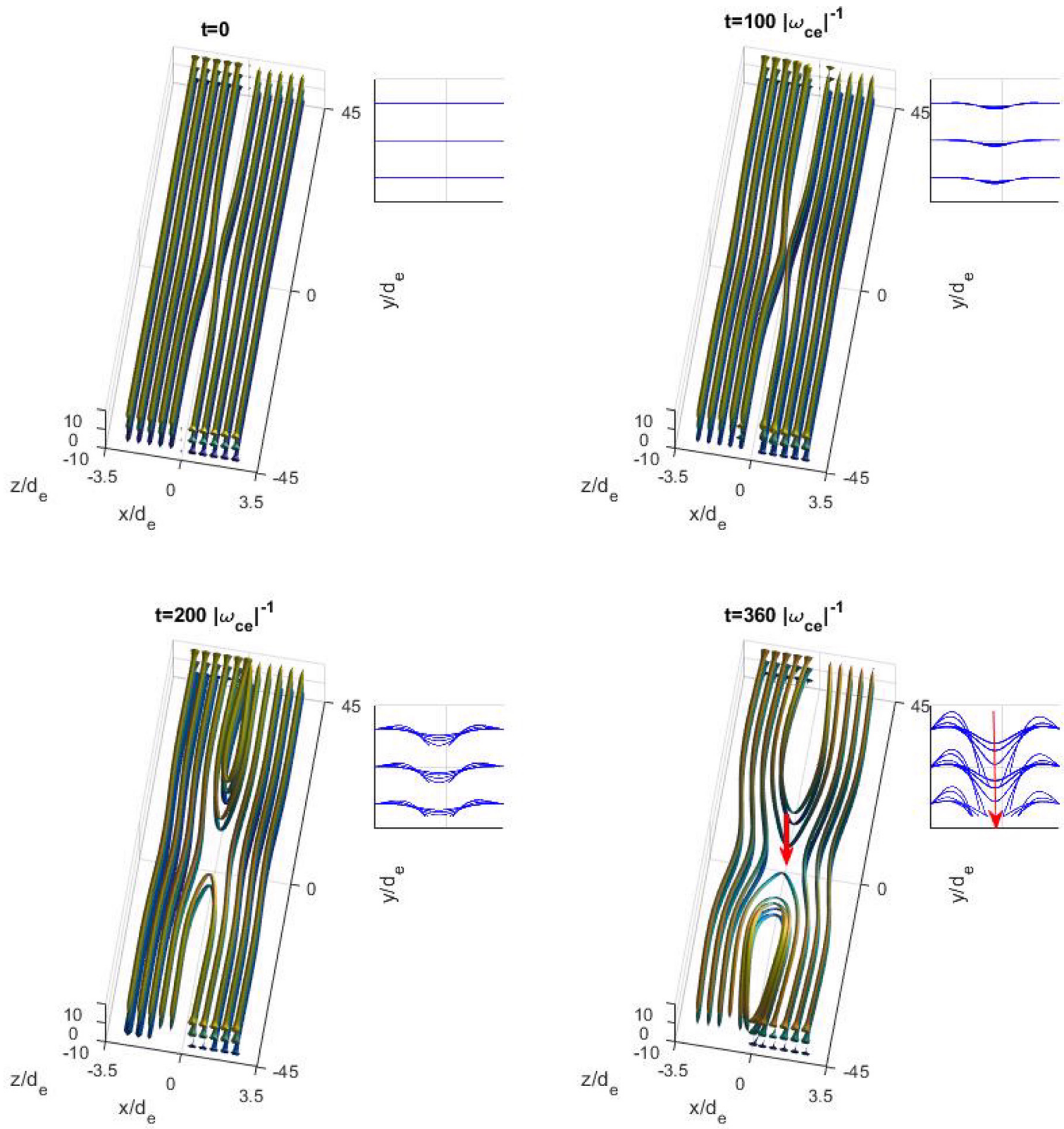

FIG. 1. Time evolution of the magnetic field streamlines for a slight perturbation to the Harris current sheet. The color represents the height of the streamline location, yellow for positive and blue for negative. Some streamlines are cut off as they exit the grid. The field of view is zoomed in for clarity of presentation. The smaller plots on the right side of each plot are the 2D streamlines in the $y-z$ plane. Clear convection of the field lines in the electron flow direction (red arrow), associated with quadrupole out-of-plane magnetic fields, is observed.

the simulated $\gamma_{s}$ for different values of $L_{x}$ and $L_{y}$. The orders of the rates agree strikingly well with each other, validating the numerical calculation by Bellan. The slight differences in number may be due to the different form of vector potential used by the author.

TABLE I. Comparisons between simulated $\gamma_{s}$ and calculated $\gamma_{c}$ for different length parameters of the current sheet.

\begin{tabular}{lccc}
\hline \hline$L_{x} / d_{e}$ & $L_{y} / d_{e}$ & $\gamma_{s} / \omega_{c e}\left(\times 10^{-3}\right)$ & $\gamma_{c} / \omega_{c e}\left(\times 10^{-3}\right)$ \\
\hline 1 & 10 & $40.19 \pm 0.40$ & 43.40 \\
2 & 10 & $17.56 \pm 0.10$ & 22.08 \\
2 & 16 & $12.95 \pm 0.50$ & 13.80 \\
5 & 16 & $5.21 \pm 0.05$ & 4.01 \\
\hline \hline
\end{tabular}

\section{B. Canonical circulation and reconnection picture}

Figure 2 shows the time evolution of a cross section in the $x-y$ plane. The contours are the out-of-plane magnetic fields, red arrows are electron flow vectors, and the streamlines are the in-plane magnetic fields. The result agrees with previous $2 \mathrm{D}$ simulations and experiments ${ }^{21,22,38,39}$ in the following aspects: electron inflow in the $\pm x$ directions, fast electron outflow in the $\pm y$ directions, generation of quadrupole out-of-plane magnetic fields, and a narrow central electron current. The quadrupole magnetic fields are consistent with the electron inflow and outflow directions.

Figure 3(a) shows canonical circulation flux tubes (i.e., Q) at $260\left|\omega_{c e}\right|^{-1}$. This specific time was chosen so that the flux tubes do not exit the grid. They pile up in the $x-y$ plane near $x=0$ due to electron inflow towards this region, but do 

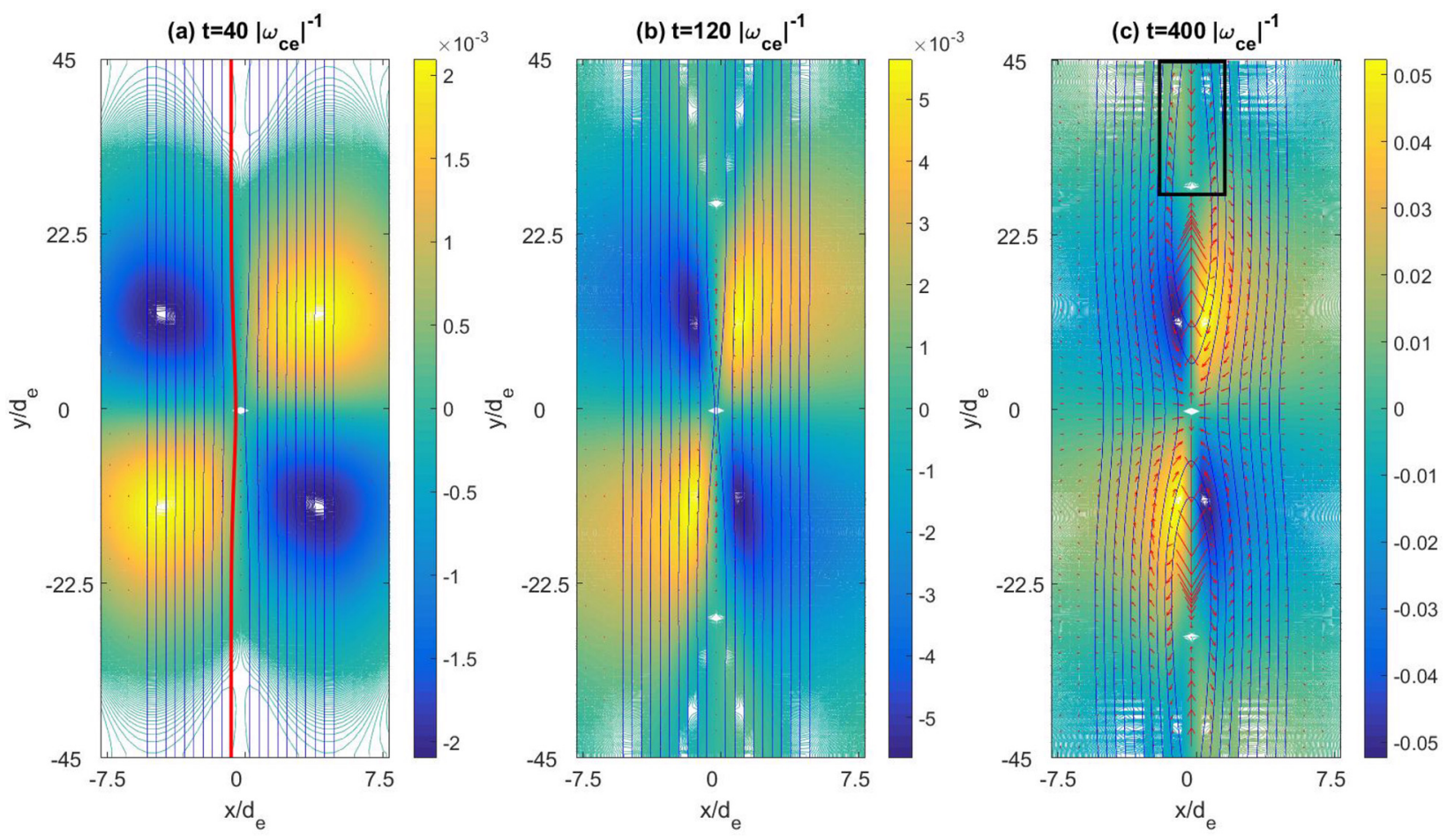

FIG. 2. 2D cross sections of the reconnection process at times (a) $40\left|\omega_{c e}\right|^{-1}$ (b) $120\left|\omega_{c e}\right|^{-1}$ (c) $400\left|\omega_{c e}\right|^{-1}$. The contours are the out-of-plane magnetic fields, red arrows are electron flow vectors, and the streamlines are the in-plane magnetic fields.

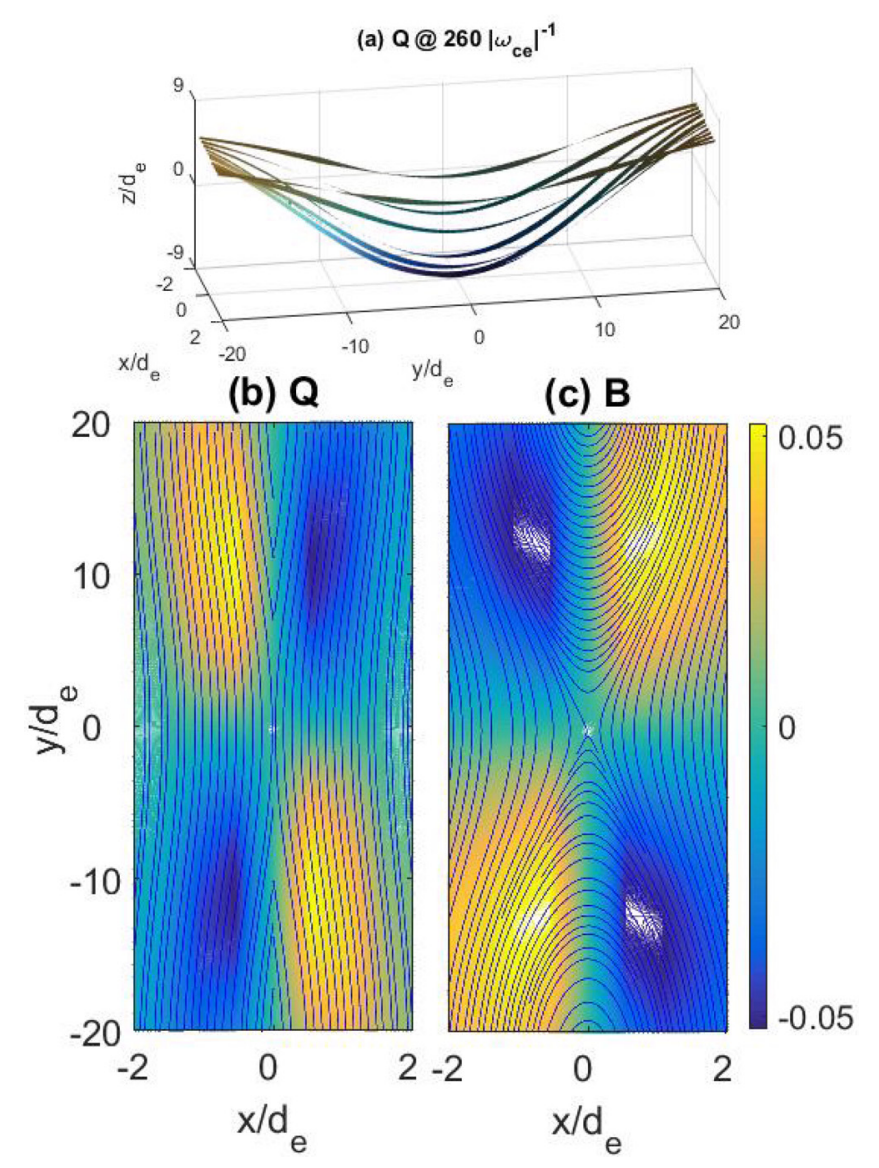

FIG. 3. (a) Canonical circulation (Q) flux tubes at $260\left|\omega_{c e}\right|^{-1}$. They accumulate around $x=0$, but do not reconnect (up to numerical round-off errors). They instead convect downwards with the electron current sheet in the middle. Corresponding 2D cross-sections for (b) $\mathbf{Q}$ and (c) $\mathbf{B}$. The colored contours are the out-of-plane components and the streamlines are the in-plane components. not reconnect (up to numerical round-off errors). They instead convect downwards with the electron current sheet location on the $y$-axis. This is more evident in Fig. 3(b) which shows the in-plane (streamlines) and out-of-plane (colored contours) components of $\mathbf{Q}$. Both the in-plane components (streamlines) and the out-of-plane components (contours) of $\mathbf{Q}$ pile-up around the center, both accordingly growing in time (see also Fig. 4 of Ref. 15 and Ref. 18). Figure 3(c) shows the corresponding plot of $\mathbf{B}$ for comparison; the $\mathbf{B}$ fields reconnect and convect away from the $\mathrm{X}$ point in the $\pm y$ and $-z$ directions instead of piling up.

This piling-up can be seen mathematically by first rearranging Eq. (9) as follows:

$$
\frac{\partial \mathbf{Q}}{\partial \bar{t}}=\bar{\nabla} \times(\overline{\mathbf{u}} \times \mathbf{Q})=\mathbf{Q} \cdot \bar{\nabla} \overline{\mathbf{u}}-\overline{\mathbf{u}} \cdot \bar{\nabla} \mathbf{Q},
$$

which can be expressed using the convective derivative as

$$
\frac{D \mathbf{Q}}{D \bar{t}}=\mathbf{Q} \cdot \bar{\nabla} \overline{\mathbf{u}}=\mathbf{Q}_{\perp} \cdot \bar{\nabla}_{\perp} \overline{\mathbf{u}},
$$

where $\partial / \partial \bar{z}=0$ was used. In order to evaluate $Q_{x}$, we first note that $\left(u_{x}\right)_{x=0}=0$ and $\left(\partial u_{x} / \partial y\right)_{x=0}=0$ because $u_{x}$ is antisymmetric with respect to $x$, and symmetric with respect to $y$. We then note that $\left(Q_{x}\right)_{x=0, t=0}=\left(\partial u_{z} / \partial y-B_{x}\right)_{x=0, t=0}=0$ because of the assumed initial condition that $\mathbf{B}$ is vertical everywhere and that $u_{z}$ is symmetric with respect to $y$. Therefore, at $x=0$

$$
\frac{D Q_{x}}{D \bar{t}}=\mathbf{Q}_{\perp} \cdot \bar{\nabla}_{\perp} \bar{u}_{x}=Q_{x} \frac{\partial \bar{u}_{x}}{\partial \bar{x}}+Q_{y} \frac{\partial \bar{u}_{x}}{\partial \bar{y}}=0,
$$

so $\left(Q_{x}\right)_{x=0}=0$ at all times. This is also obvious since finite $\left(Q_{x}\right)_{x=0}$ would imply a reconnection-like crossing of $\mathbf{Q}$ from 
$x<0$ to $x>0$, which is forbidden by the frozen-in condition and inflow stagnation at $x=0$.

We now turn attention to examination of $Q_{y}$ near $x=0$. The $y$ component of Eq. (18) can be written

$$
\frac{D Q_{y}}{D \bar{t}}=\mathbf{Q}_{\perp} \cdot \bar{\nabla}_{\perp} \bar{u}_{y}=Q_{x} \frac{\partial \bar{u}_{y}}{\partial \bar{x}}+Q_{y} \frac{\partial \bar{u}_{y}}{\partial \bar{y}} .
$$

However, we have shown that $\left(Q_{x}\right)_{x=0}=0$, so near the $y$ axis Eq. (20) becomes

$$
\left(\frac{D Q_{y}}{D \bar{t}}\right)_{x \approx 0}=\left(Q_{y}\right)_{x \approx 0}\left(\frac{\partial \bar{u}_{y}}{\partial \bar{y}}\right)_{x \approx 0} .
$$

Here, the quadrupole magnetic fields near the $\mathrm{x}$-point go like $B_{z} \sim \alpha(t) x y$ where $\alpha(t)$ is positive, so $u_{y} \sim \partial B_{z} / \partial x \sim \alpha(t) y$. Thus, $\partial u_{y} / \partial y \sim \alpha(t)>0$, so Eq. (21) shows that $Q_{y}$ will grow exponentially with time as

$$
\left(Q_{y}\right)_{x \approx 0, t} \approx\left(Q_{y}\right)_{x \approx 0, t=0} \exp \left(\int_{0}^{\bar{t}} \frac{\partial \bar{u}_{y}}{\partial \bar{y}} d \bar{t}^{\prime}\right) .
$$

This shows that the magnitude of $Q_{y}$ near the $y$-axis (but not exactly at $x=0$ as $Q_{y}$ is antisymmetric about the $y$-axis) increases exponentially in time with a growth rate $\gamma \approx \partial \bar{u}_{y} / \partial \bar{y} \approx u_{y} / L_{y}$. Because incompressibility dictates that the outflow velocity satisfies $u_{y} L_{x}=$ const., this gives a growth rate scaling $\gamma \sim 1 /\left(L_{x} L_{y}\right)$ which is in agreement with the scaling calculated in Ref. 23 .

Because $\mathbf{Q}$ flux tubes not only pile up but are also stretched in the $z$-direction, $Q_{z}$ must also exhibit exponential behavior. Figure 4 shows a log plot of the time dependence of $Q_{z}$ and the two terms constituting $Q_{z}$ at the location $x=d_{e}$, $y=2 d_{e}$. Exponential growth in the latter parts of the process can clearly be seen, as in Eq. (22). It can also be seen that near $t=100\left|\omega_{c e}\right|^{-1}$ the electron vorticity term $\nabla \times \mathbf{u}$ overcomes the magnetic field term $\mathbf{B}$ and becomes the dominant term in $\mathbf{Q}$; this corresponds to a conversion of magnetic field

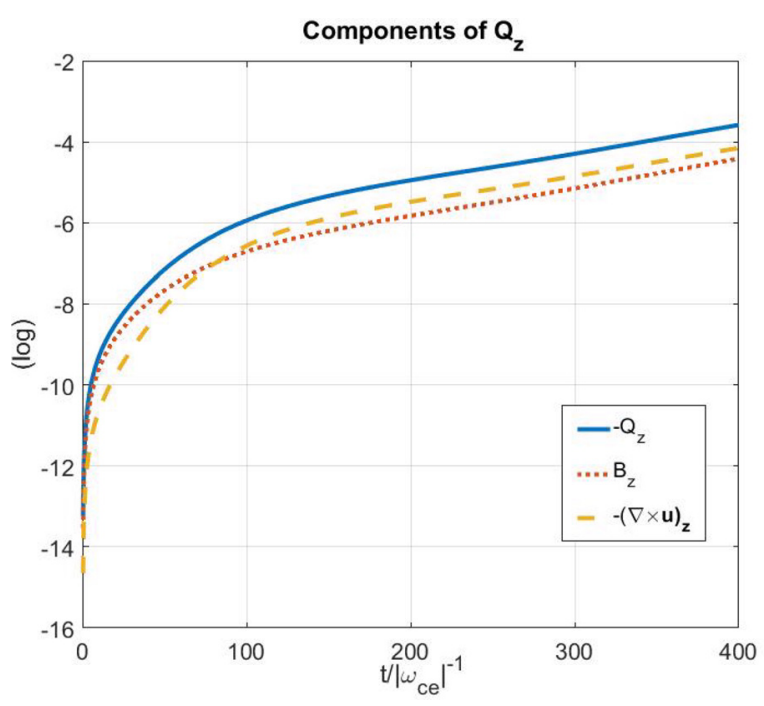

FIG. 4. Log plot of the two components of $Q_{z}$ at $x=1 d_{e}$ and $y=2 d_{e}$ as a function of time. The electron vorticity overcomes the magnetic field as the reconnection progresses. Eventual exponential growth is also seen. into electron flow. Combining this with the exponential growth of the magnitude of $\mathbf{Q}$, we can attribute the huge electron outflow acceleration associated with reconnection to the exponential increase of the relevant electron vorticity $\nabla \times \mathbf{u}$ near the conversion region.

The exponential increase of $\mathbf{Q}$ together with the conservation of canonical circulation flux $C=\int \mathbf{Q} \cdot d \mathbf{s}$ means that the cross-section of a $\mathbf{Q}$ flux tube must become exponentially smaller with time as the magnitude of $\mathbf{Q}$ increases. On the other hand since $\mathbf{Q}$ is frozen to the electron fluid and the electron fluid is incompressible, the volume of a $\mathbf{Q}$ flux tube must remain constant. Since the cross-section of the $\mathbf{Q}$ flux tube is decreasing in time, in order for the volume of this flux tube to remain constant, the $\mathbf{Q}$ flux tube must become longer which corresponds to the dipping and stretching of the $\mathbf{Q}$ flux tubes. This is analogous to a rubber band whose radius decreases as it is stretched. This also explains how the decrease in $\mathbf{B}$ field gives an exponential increase in electron velocity, since $C=\int \nabla \times \mathbf{u} \cdot d \mathbf{s}-\int \mathbf{B} \cdot d \mathbf{s}=\oint \mathbf{u} \cdot d \mathbf{l}-\int \mathbf{B} \cdot d \mathbf{s} ;$ so, as $\mathbf{B} \rightarrow$ 0 and $d \mathbf{l} \rightarrow 0$ near the conversion region, $\mathbf{u} \rightarrow \infty$.

Figures 5(a) and 5(b) visually demonstrate the stretching and thinning of a flux tube. As it stretches, the tube gets thinner in the $x-y$ plane, especially in the region where the quadrupole fields are present $\left(y \approx \pm 10 d_{e}\right)$. At $y=0$, the tube is slightly thicker because $Q_{x}=Q_{z}=0$ at this point, but as shown in Figure 5(c), $Q_{y}$ clearly grows in time near the conversion region. The tube does not compress in the $y-z$ plane because of the $\partial / \partial z \rightarrow 0$ requirement in $2 \mathrm{D}$. Also note the helical evolution of the flux tube; this is related to whistler wave propagation, as will be shown in Sec. III D.

The fact that $\mathbf{Q}$ is the sum of the normalized fluid vorticity and the magnetic field is important because it can give simple explanations for several phenomena. For example, the spiked central electron current filaments that change spatially over $d_{e}$ scales in this regime $e^{15,16,18,24}$ may be explained as follows: as shown above, $\left|\left(Q_{y}\right)_{x \approx 0}\right| \rightarrow \infty$ and $\left(B_{y}\right)_{x=0}=0$

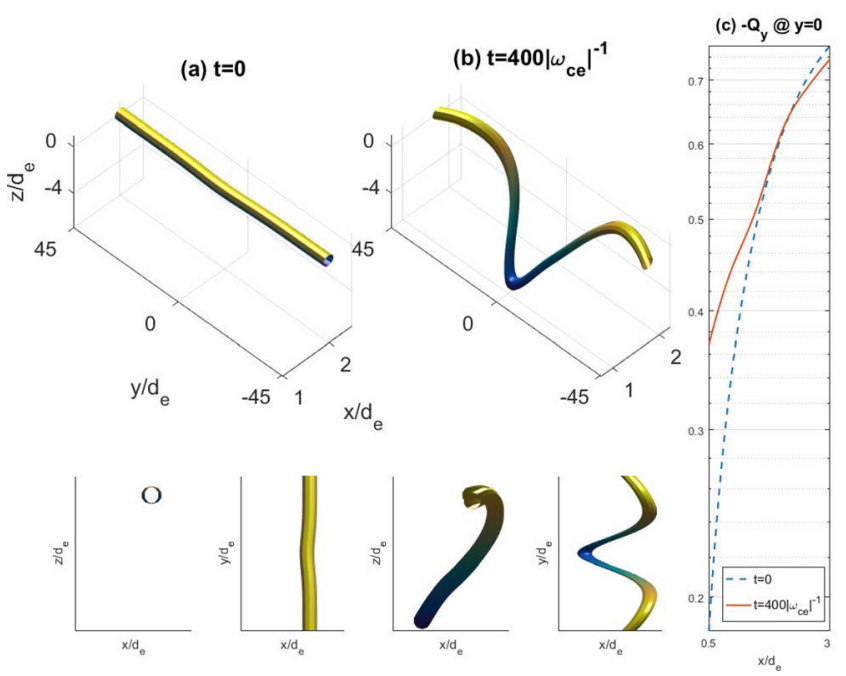

FIG. 5. A $\mathbf{Q}$ flux tube in the $x>0$ region at (a) $\mathrm{t}=0$ and (b) $400\left|\omega_{c e}\right|^{-1}$. As the flux tube is stretched downwards along with the electron current, its radius decreases, so the magnitude of $\mathbf{Q}$ increases. The color represents the height in the $z$-direction. (c) The time evolution of $Q_{y}$ at $y=0$. Near $x=0$, $Q_{y}$ increases over time. 
by antisymmetry about the $y$-axis, but $Q_{y}=-\frac{\partial u_{z}}{\partial x}-B_{y}$ so $\left|\left(\frac{\partial u_{z}}{\partial x}\right)_{x=0}\right| \rightarrow \infty$. Therefore, the central current density does not have a "plateau," but is rather "spiked" along the $x$-direction, since a plateau would mean that $\left(\frac{\partial u_{z}}{\partial x}\right)_{x=0}=0$ no matter how big $u_{z}$ is.

Combining all these results, we can construct an intuitive picture for two-fluid reconnection:

1. The initial state has straight $\mathbf{Q}$ field lines (dominantly $\mathbf{B}$ ) pointing in the positive $y$ direction in the $x>0$ region and in the negative $y$ direction in the $x<0$ region convecting downwards with negative $z$ velocity that is the same as the electron downward flow velocity.

2. A current perturbation localized in the $x$ and $y$ directions makes some regions of a $\mathbf{Q}$ flux tube approach the central current (as in Figure 5(a)). In most reconnection situations, initial field lines are convex with respect to the center (e.g., X-type configurations ${ }^{41}$ ), so this is the typical initial state.

3. The central electron current is stronger near $x=0$, so the flux tube in this region convects faster downwards than in other regions. Since $\mathbf{Q}$ field lines cannot break, the central current "plucks" the flux tube, making a "bump" in the -z-direction near the localized region, as in Figure 5(b)just like a plucked guitar string. This makes the out-ofplane, quadrupole fields in the $x-y$ plane.

4. The quadrupole fields correspond to electron inflow towards $x=0$ (since $u_{x}=-\partial B_{z} / \partial y$ ), so the $\mathbf{Q}$ field lines are carried further towards a conversion region of a few electron skin depth scales.

5. At this scale, the magnetic field converts to electron vorticity while preserving total $\mathbf{Q}$ flux (which has become dominantly $\oint \mathbf{u} \cdot d \mathbf{l}$ ), so the magnetic fields reconnect while the electrons are accelerated in the outflow directions.

6. As the $x-y$ projections of the $\mathbf{Q}$ field lines pile up near $x=0$, and the $y$-midpoint of the $\mathbf{Q}$ field lines convect further downwards in the $-z$-direction, $\mathbf{Q}$ flux tubes are stretched, and the magnitudes of $\mathbf{Q}$ and $\nabla \times \mathbf{u}$ in particular increase exponentially because the stretching reduces the cross-sectional area of the incompressible $\mathbf{Q}$ flux tubes.

7. Since the electron inflow carries more $\mathbf{Q}$ field lines from further away towards the conversion region, the entire process has positive feedback, so it continues until all particles are exhausted via the outflow. Additional evidence that the process is unstable rather than wave-like is in Refs. 42-44, where the reconnection process was found to relax to another macroscopic equilibrium rather than "bounce back."

\section{Energy and helicity}

Figure 6 shows the time evolution of the energy components in Eq. (13). The quantities were integrated not over the entire volume, but over the main region of interest $\left(-3 d_{e}<x<3 d_{e},-20 d_{e}<y<20 d_{e}\right)$. This is to minimize the numerical error that arises from the dominance of the

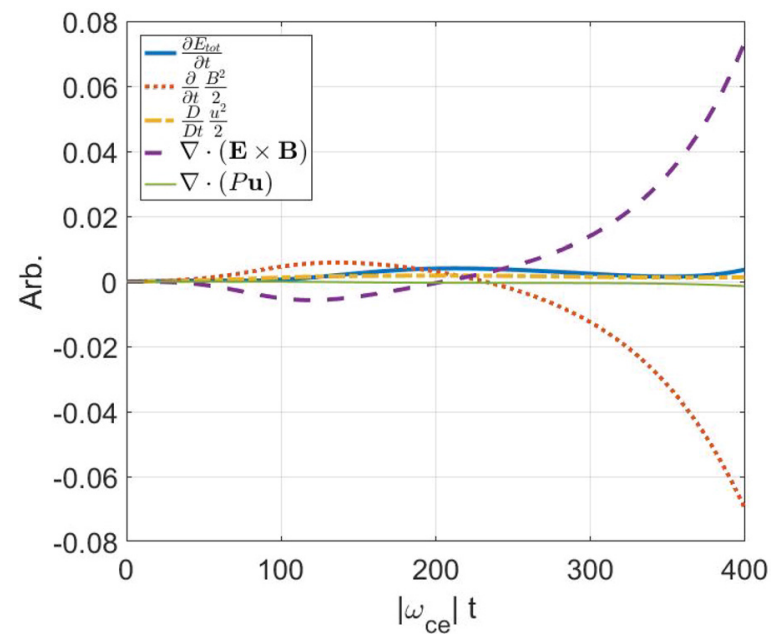

FIG. 6. Time evolution of various energy components as a function of time.

magnetic energy density in the initial configuration. The total energy was well conserved within a $5 \%$ error.

It is evident from the plot that the magnetic energy initially increases due to the incoming Poynting flux towards the $\mathrm{x}$-point. Then, at around 100 to $200\left|\omega_{c e}\right|^{-1}$, magnetic energy starts to decay by converting into both electron kinetic energy and Poynting flux, with the latter being dominant. Since, as seen in Eq. (16), whistler waves represent waves in this regime, they are the main carriers of energy downstream.

Total canonical helicity was conserved in such a way that the helicity densities exhibited a quadrupole nature, similar to the quadrupole magnetic fields. Figure 7 shows the maximum values of the four canonical helicity density components described in Eq. (14) as a function of time. At earlier times, magnetic helicity $\mathbf{A} \cdot \mathbf{B}$ is dominant, but as reconnection progresses, the $\mathbf{A} \cdot \nabla \times \mathbf{u}$ term becomes dominant. The sudden jump around $t \sim 50\left|\omega_{c e}\right|^{-1}$ is because the helicity density around the conversion region increases more rapidly than that outside it. This shows indeed that sole analysis of magnetic helicity is not sufficient to describe the entire reconnection process. ${ }^{13,25}$

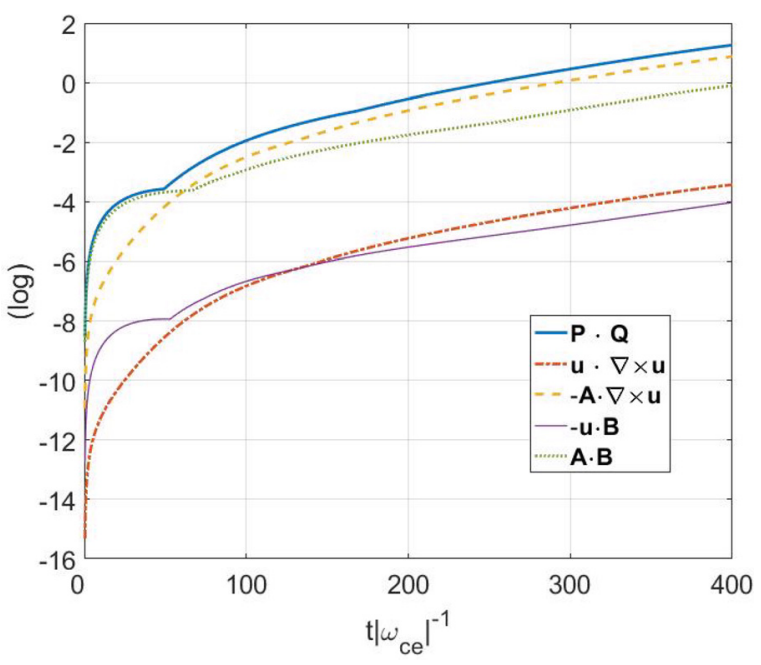

FIG. 7. Log plot of maximum values of different helicity components. 


\section{Whistler wave association}

One aspect to note in particular is the generation of additional out-of-plane magnetic fields in the outflow region (alternating colors in black box in Figure 2(c)). These were also frequently observed by other simulations, ${ }^{21,22,45}$ but were not discussed in detail. These fields may play a role in opposing the electron outflow and instigating whistler waves in the $\pm y$ directions. Their having opposite parities from the quadrupole field around the x-point supports this idea, as it hints to wave-like behavior.

To further investigate this concept, the temporal evolution of one of the reconnected magnetic field lines (e.g., red line in Figure 2(a)) is examined in detail with particular attention to the role of this out-of-plane $B_{z}$ (black box in Figure 2(c)) in the latter parts of the process. This is shown in Figure 8 over the time interval 0 to $180\left|\omega_{c e}\right|^{-1}$ in steps of $20\left|\omega_{c e}\right|^{-1}$. The bottom subfigure shows the final field line with respect to the straightened-out initial field line. The A, $\mathrm{B}$, and $\mathrm{Cs}$ in both subfigures represent the same locations along the initial field line.

It is seen that the field becomes helical with respect to the initial field state. In the negative $\mathrm{x}$ region, the propagation

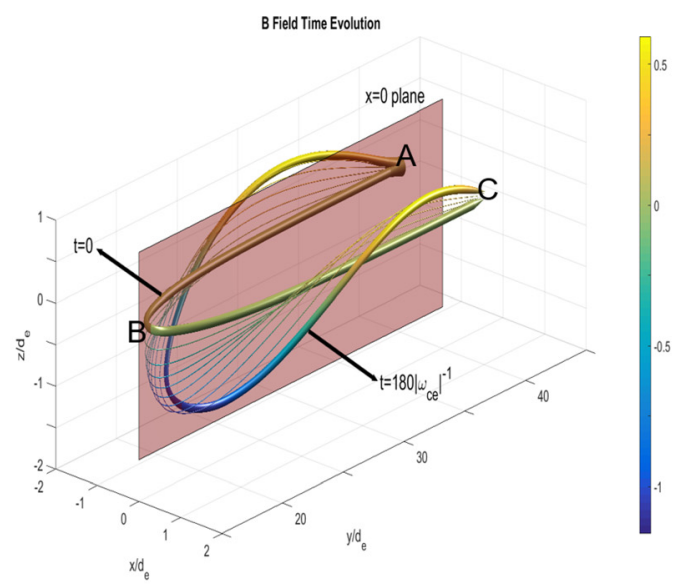

(a)

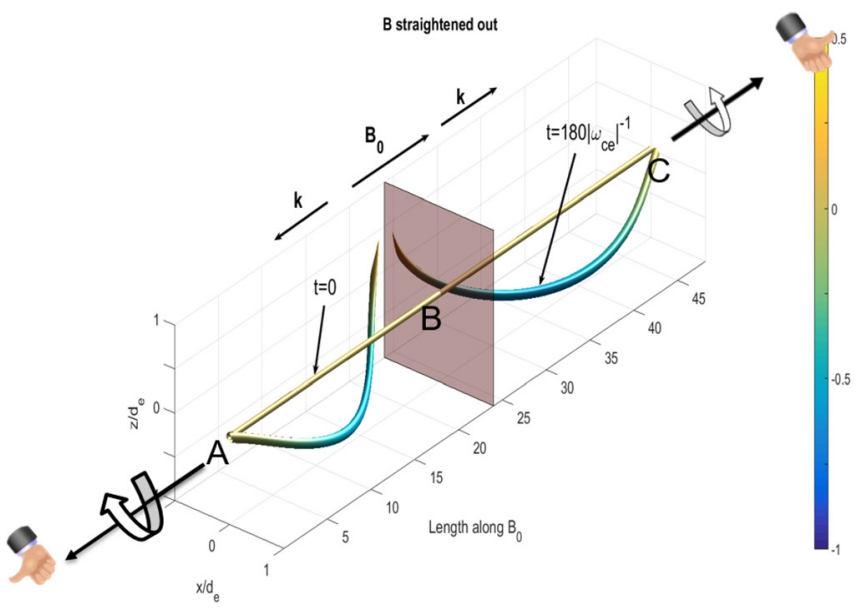

(b)

FIG. 8. Temporal evolution of one of the reconnected magnetic field lines (e.g., red line in Figure 2). The bottom figure shows the same field lines when the initial field line is straighted out. direction (mainly $+y$ ) is against the background field (mainly $-y$ ), so the field helicity is spatially right-handed, and similarly in the positive $x$ region, the propagation direction (mainly $+y$ ) is along the background field (mainly $+y$ ), so the field helicity is spatially left-handed as it should be. ${ }^{46}$ If we assume that the initial field line $(t=0)$ is the background field and that the final field line $\left(t=180\left|\omega_{c e}\right|^{-1}\right)$ results from a perturbation to this background at the transition region, the final state is consistent with whistler wave propagation towards the outflow region. The fact that the central perturbation strength is about twice the propagated perturbation supports this idea. Helical evolution is also demonstrated in Figure 5(b).

One can imagine that when the reconnected field line relaxes back to its minimum energy state, the helical tension will be propagated away from the source as whistler waves along the reconnected field lines. The frequency of this wave is guaranteed to be in the whistler regime since the reconnection rate was found to be of this order.

This concept agrees very well with recent spacecraft measurements ${ }^{47-50}$ near the Earth's magnetosheath and magnetospheric regions, where strong quasi-parallel whistler waves were observed at the outflow region. Some authors in particular state that the electron diffusion region may be the source, because they observed whistler waves propagating away from the $x$-point to the outflow direction along the magnetic field [Refs. 47 and 50, Figure 1]. Although the above authors observed whistler waves near the separatrix region propagating towards the region as well, these were generally attributed to electron beam-driven whistler instabilities or other kinetic effects, which are impossible to reproduce in this simulation.

It is important to clarify some properties of whistler waves regarding polarization and helicity. Whistler waves propagate mainly along the background magnetic field $\mathbf{B}_{0}$ at a slightly oblique angle (quasi-parallel) while maintaining circular polarization ${ }^{51}$ (the $\cos \theta$ in the dispersion relationEq. (16)—prevents perpendicular propagation). Regarding this circular polarization, one has to be careful when characterizing the handedness of the field helicity, since righthanded circular polarization of the wave only makes sense temporally when looking against the $\mathbf{B}_{0}$ direction; the polarization vector always rotates in time in a right-handed sense regardless of the propagation direction. However, the field helicity is spatially left-handed for propagation along $\mathbf{B}_{0}$ and right-handed for propagation against $\mathbf{B}_{0}$ (see detailed analysis by Ref. 46). Therefore, one has to be careful when identifying spatial right-handed circular polarization as whistler waves, as the direction of propagation must be specified with respect to $\mathbf{B}_{0}$.

In Ref. 23 the observed magnetic reconnection was found to be purely growing, i.e., scaling as $\exp (\gamma t)$ with no propagating waves, whereas in the present paper, propagating whistler waves are observed. There are two possible resolutions to this seeming contradiction. The first possibility is that in Ref. 23 it was assumed that the perturbation was periodic in the $y$ direction, scaling as $\sin \left(k_{y} y\right)$, whereas here there is a single perturbation centered about $x=0$ and $y=0$ with no periodicity in the $y$ direction. When the perturbation 
is periodic in the $y$ direction, the possibility of waves propagating in the $y$ direction "out of the system" is effectively prohibited because there is no preferred sense for these waves, i.e., symmetry in $y$ means the waves have no reason to be propagating either up or down, so the only possibility is that there are no waves (or standing waves which, as evidenced by finite Poynting flux out of the system, were not observed). The second possibility is that the wave is a non-linear phenomenon which the simulation can resolve but the linear theory cannot.

\section{CONCLUSION}

We have presented a generalized picture of $2 \mathrm{D}$ nondriven magnetic reconnection in the collisionless two-fluid regime. Rearranging two-fluid equations gives the frozen-in condition of the canonical circulation flux into the electron velocity. Solving this condition in the reconnection geometry yields the quadrupole out-of-plane magnetic fields and an exponential increase in the magnitude of $\mathbf{Q}$ near the reconnection region due to stretching of the $\mathbf{Q}$ flux tubes by the central electron current. Because $\mathbf{Q}$ consists of two terms - the magnetic field $\mathbf{B}$ and the electron vorticity $\nabla \times \mathbf{u}-$ and the latter dominates while conserving the flux $C=\int \mathbf{Q} \cdot d \mathbf{s}$ in the conversion region, the magnetic field lines reconnect and the electrons are accelerated. The rate was found to agree well with a previous calculation. ${ }^{23}$ Simple explanations to some phenomena related to reconnection in this regime, such as spiked electron current filaments and fast reconnection rates, were also given. It was also found that the magnetic energy is propagated mainly in the form of the Poynting flux, and that the canonical helicity $\int \mathbf{P} \cdot \mathbf{Q} d V$ as a whole must be considered rather than solely the magnetic helicity. Finally, a mechanism for whistler wave generation and propagation was proposed in relation to past observations.

Since the computer code was written in full threedimensions with the ultimate goal of investigating 3Dlocalized reconnections, future work will be focussed on how the geometry of the $\mathbf{Q}$ flux tubes changes in the $3 \mathrm{D}$ regime and how its constituents behave accordingly. It is expected that the $\mathbf{Q}$ flux tubes will "twist" near a 3D-localized point and in effect increase the magnitude of $\mathbf{Q}$.

\section{ACKNOWLEDGMENTS}

This material is based upon work supported by the National Science Foundation under Award No. 1059519, by the Air Force Office of Scientific Research under Award No. FA9550-11-1-0184, and by the U.S. Department of Energy
Office of Science, Office of Fusion Energy Sciences under Award No. DE-FG02-04ER54755.

\section{APPENDIX: ANALYTICAL SOLUTION FOR CURRENT- RING-INDUCED WHISTLER WAVES}

Let us examine the relevant linearized two-fluid equations with a Dirac-delta oscillating current ring of radius $a$ with frequency $\omega$ and magnitude $I$ in the $\hat{\phi}$ direction. The tilde signifies the linearized quantities.

$$
\begin{gathered}
\nabla \times \tilde{\mathbf{E}}=-\frac{\partial \tilde{\mathbf{B}}}{\partial t}, \\
\nabla \times \tilde{\mathbf{B}}=\mu_{0} \tilde{\mathbf{J}}, \\
\tilde{\mathbf{J}}=n_{e} q_{e} \tilde{\mathbf{u}}_{e}+I \delta(r-a) \delta(z) \hat{\phi}, \\
\frac{m_{e}}{q_{e}} \frac{\partial \tilde{\mathbf{u}}_{e}}{\partial t}=\tilde{\mathbf{E}}+\tilde{\mathbf{u}}_{e} \times \mathbf{B}_{0},
\end{gathered}
$$

where the convective derivative has been reduced to the partial derivative since $\tilde{\mathbf{u}}_{e} \cdot \nabla \tilde{\mathbf{u}}_{e}$ is of second order. Assuming oscillations $\sim e^{-i \omega t}$

$$
\begin{gathered}
\nabla \times \tilde{\mathbf{E}}=i \omega \tilde{\mathbf{B}} \\
\tilde{\mathbf{E}}=-i \omega \frac{m_{e}}{q_{e}} \tilde{\mathbf{u}}_{e}-B_{z} \tilde{\mathbf{u}}_{e} \times \hat{z} .
\end{gathered}
$$

Doing a similar calculation to that in Section II and normalizing, we have

$$
\begin{aligned}
\bar{\nabla} & \times([\bar{\nabla} \times \tilde{\mathbf{B}}] \times \hat{z})+i \bar{\omega}\left(\bar{\nabla}^{2} \tilde{\mathbf{B}}-\tilde{\mathbf{B}}\right) \\
& =\mu_{0} I \frac{\omega_{p e}}{c}\left(\begin{array}{c}
\delta(\bar{r}-\bar{a}) \delta^{\prime}(\bar{z})[\hat{\phi}+i \bar{\omega} \hat{r}] \\
-i \bar{\omega}\left[\frac{\delta(\bar{r}-\bar{a}) \delta(\bar{z})}{\bar{r}}+\delta^{\prime}(\bar{r}-\bar{a}) \delta(\bar{z})\right] \hat{z}
\end{array}\right) .
\end{aligned}
$$

Since $\bar{\omega}=\omega / \omega_{c e} \ll 1$ in the whistler regime, this means that a current ring acts mainly as a azimuthal source of $\tilde{\mathbf{B}}$ in the plasma.

Fourier transforming and decomposing the equation in the $r, \phi$ and $z$ directions give

$$
\begin{gathered}
\left.-\bar{k}_{z} \bar{k}_{\perp} B_{\phi}-i \bar{\omega}\left(\bar{k}_{z}^{2}+\bar{k}_{\perp}^{2}+1\right) B_{z}=-\alpha i \bar{\omega} \bar{k}_{\perp} \bar{a} J_{1}\left(\bar{k}_{\perp} \bar{a}\right), \quad \text { (A } 8\right) \\
\bar{k}_{z}^{2} B_{\phi}-i \bar{\omega}\left(\bar{k}_{z}^{2}+\bar{k}_{\perp}^{2}+1\right) B_{r}=-\alpha \bar{\omega} \bar{k}_{z} \bar{a} J_{0}\left(\bar{k}_{\perp} \bar{a}\right), \quad \text { (A9) } \\
-\bar{k}_{z}^{2} B_{r}+\bar{k}_{z} \bar{k}_{\perp} B_{z}-i \bar{\omega}\left(\bar{k}_{z}^{2}+\bar{k}_{\perp}^{2}+1\right) B_{\phi}=\alpha i \bar{k}_{z} \bar{a} J_{0}\left(\bar{k}_{\perp} \bar{a}\right),
\end{gathered}
$$

where $\alpha=2 \pi \mu_{0} I \frac{\omega_{p e}}{c}$. Solving for $\tilde{\mathbf{B}}\left(\bar{k}_{\perp}, \bar{k}_{z}, \bar{\omega}\right)$,

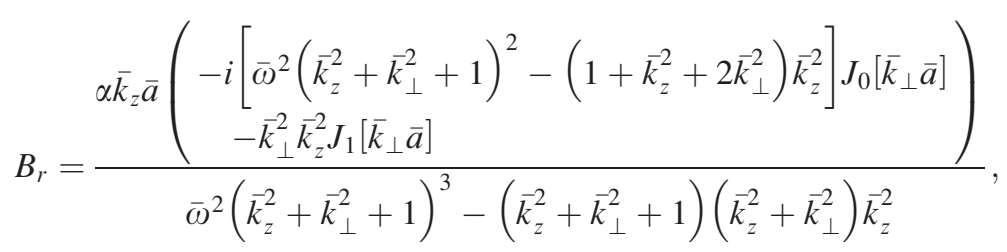




$$
\begin{gathered}
B_{\phi}=\frac{-\alpha \bar{k}_{z} \bar{a} \bar{\omega}\left(\left[1+\bar{k}_{\perp}^{2}\right] J_{0}\left[\bar{k}_{\perp} \bar{a}\right]+i \bar{k}_{\perp}^{2} J_{1}\left[\bar{k}_{\perp} \bar{a}\right]\right)}{\bar{\omega}^{2}\left(\bar{k}_{z}^{2}+\bar{k}_{\perp}^{2}+1\right)^{2}-\left(\bar{k}_{z}^{2}+\bar{k}_{\perp}^{2}\right) \bar{k}_{z}^{2}}, \\
B_{z}=\frac{\alpha \bar{k}_{\perp} \bar{a}\left(\begin{array}{c}
-i\left[1+\bar{k}_{\perp}^{2}\right] \bar{k}_{z}^{2} J_{0}\left[\bar{k}_{\perp} \bar{a}\right] \\
+\left[\bar{k}_{z}^{2}(\bar{\omega}-1)+\left(1+\bar{k}_{\perp}^{2}\right) \bar{\omega}\right]\left[\bar{k}_{z}^{2}+\left(\bar{k}_{z}^{2}+\bar{k}_{\perp}^{2}+1\right) \bar{\omega}\right] J_{1}\left[\bar{k}_{\perp} \bar{a}\right]
\end{array}\right)}{\bar{\omega}^{2}\left(\bar{k}_{z}^{2}+\bar{k}_{\perp}^{2}+1\right)^{3}-\left(\bar{k}_{z}^{2}+\bar{k}_{\perp}^{2}+1\right)\left(\bar{k}_{z}^{2}+\bar{k}_{\perp}^{2}\right) \bar{k}_{z}^{2}} .
\end{gathered}
$$

Setting the denominator to zero gives the correct whistler dispersion relation.

Solving $B_{\phi}$ via inverse Fourier transforms involving contour integrals, we have the final integral solution

$$
\begin{aligned}
& B_{\phi}(\bar{r}, \bar{z}, \bar{\omega}) \\
& =-\frac{\alpha \bar{a} \bar{\omega} i}{4 \pi} \int_{0}^{\infty} \bar{k}_{\perp} d \bar{k}_{\perp} \\
& \quad \times \frac{J_{0}\left[\bar{k}_{\perp} \bar{r}\right]\left(\left[1+\bar{k}_{\perp}^{2}\right] J_{0}\left[\bar{k}_{\perp} \bar{a}\right]+i \bar{k}_{\perp}^{2} J_{1}\left[\bar{k}_{\perp} \bar{a}\right]\right)}{\sqrt{\bar{k}_{\perp}^{4}+4 \bar{\omega}^{2}\left(1+\bar{k}_{\perp}^{2}\right)}}
\end{aligned}
$$

$\times \cos A \bar{z} \quad$ where

$$
A=\sqrt{\frac{2 \bar{\omega}^{2}\left(1+\bar{k}_{\perp}^{2}\right)-\bar{k}_{\perp}^{2}+\sqrt{\bar{k}_{\perp}^{4}+4 \bar{\omega}^{2}\left(1+\bar{k}_{\perp}^{2}\right)}}{2\left(1-\bar{\omega}^{2}\right)} .}
$$

${ }^{1}$ K. Shibata and T. Magara, Living Rev. Sol. Phys. 8, 45-63 (2011).

${ }^{2}$ T. G. Forbes, Nat. Phys. 9, 456 (2013).

${ }^{3}$ X. H. Deng and H. Matsumoto, Nature 410, 557 (2001).

${ }^{4} \mathrm{~J}$. Wesson, Nucl. Fusion 30, 2545 (1990).

${ }^{5}$ F. Mead, T. York, B. Jacoby, and E. Klevans, Bull. Am. Phys. Soc. 25, $882(1980)$.

${ }^{6}$ Y. Ono, M. Yamada, A. Janos, and F. Levinton, Phys. Fluids B: Plasma Phys. 3, 1452 (1991).

${ }^{7}$ K.-B. Chai, X. Zhai, and P. M. Bellan, Phys. Plasmas 23, 032122 (2016).

${ }^{8}$ Y. Ono, M. Katsurai, and M. Yamada, AIP Conf. Proc. 311, 186-193 (1994).

${ }^{9}$ M. Yamada, R. Kulsrud, and H. Ji, Rev. Mod. Phys. 82, 603 (2010).

${ }^{10}$ A. Moser, Nature 482, 379 (2012).

${ }^{11}$ X. Zhai and P. M. Bellan, Phys. Plasmas 23, 032121 (2016).

${ }^{12}$ P. M. Bellan, Spheromaks (World Scientific, 2000).

${ }^{13}$ S. You, Phys. Plasmas 19, 092107 (2012).

${ }^{14}$ S. Bulanov, F. Pegoraro, and A. Sakharov, Phys. Fluids B: Plasma Phys. 4, 2499 (1992)

${ }^{15}$ D. Biskamp, E. Schwarz, and J. F. Drake, Phys. Plasmas 4, 1002 (1997).

${ }^{16}$ F. Porcelli, D. Borgogno, F. Califano, D. Grasso, M. Ottaviani, and F. Pegoraro, Plasma Phys. Controlled Fusion 44, B389 (2002).

${ }^{17}$ N. Attico, F. Califano, and F. Pegoraro, Phys. Plasmas 7, 2381 (2000).

${ }^{18}$ M. Ottaviani and F. Porcelli, Phys. Rev. Lett. 71, 3802 (1993).

${ }^{19}$ E. Cafaro, D. Grasso, F. Pegoraro, F. Porcelli, and A. Saluzzi, Phys. Rev. Lett. 80, 4430 (1998).

${ }^{20}$ A. Kingsep, K. Chukbar, and V. Yan'kov, in Reviews of Plasma Physics edited by B. Kadomtsev (Consultants Bureau, New York, 1990), Vol. 16, p. 143.

${ }^{21}$ J. F. Drake, M. A. Shay, and M. Swisdak, Phys. Plasmas 15, 042306 (2008).

${ }^{22}$ K. Fujimoto and R. D. Sydora, Geophys. Res. Lett. 35, 119112, doi:10.1029/2008GL035201 (2008).
${ }^{23}$ P. M. Bellan, Phys. Plasmas 21, 102108 (2014).

${ }^{24}$ J. Drake, D. Biskamp, and A. Zeiler, Geophys. Res. Lett. 24, 2921, doi:10.1029/97GL52961 (1997).

${ }^{25}$ S. You, Plasma Phys. Controlled Fusion 56, 064007 (2014).

${ }^{26}$ D. Del Sarto, F. Califano, and F. Pegoraro, Phys. Plasmas 12, 012317 (2005).

${ }^{27}$ N. Attico, F. Califano, and F. Pegoraro, Phys. Plasmas 8, 16 (2001).

${ }^{28}$ B. N. Kuvshinov, E. Westerhof, T. J. Schep, and M. Berning, Phys. Lett. A 241, 287 (1998).

${ }^{29}$ E. G. Harris, Il Nuovo Cimento 23, 115 (1962).

${ }^{30}$ P. M. Bellan, Phys. Plasmas 5, 3081 (1998).

${ }^{31}$ P. M. Bellan, Fundamentals of Plasma Physics (Cambridge University Press, 2008).

${ }^{32}$ P. Bellan, Phys. Fluids B: Plasma Phys. 5, 1955 (1993).

${ }^{33}$ M. E. Mandt, R. E. Denton, and J. F. Drake, Geophys. Res. Lett. 21, 73, doi:10.1029/93GL03382 (1994).

${ }^{34}$ S. E. Koonin, Computational Physics (Addison-Wesley Pub. Co., Menlo Park, California, 1986)

${ }^{35}$ R. L. Stenzel and J. M. Urrutia, Phys. Rev. Lett. 114, 205005 (2015).

${ }^{36}$ R. K. Fisher and R. W. Gould, Phys. Rev. Lett. 22, 1093 (1969).

${ }^{37}$ R. Gendrin, Planet. Space Sci. 5, 274 (1961).

${ }^{38}$ M. Yamada, J. Yoo, J. Jara-Almonte, H. Ji, R. M. Kulsrud, and C. E. Myers, Nat. Commun. 5, 4774 (2014).

${ }^{39}$ B. N. Rogers, R. E. Denton, J. F. Drake, and M. A. Shay, Phys. Rev. Lett. 87, 195004 (2001).

${ }^{40}$ D. Biskamp, Magnetic Reconnection in Plasmas (Cambridge University Press, 2005), Vol. 3.

${ }^{41}$ I. J. Craig and A. McClymont, Astrophys. J. 371, L41 (1991).

${ }^{42}$ D. Grasso, F. Califano, F. Pegoraro, and F. Porcelli, Phys. Rev. Lett. 86, 5051 (2001)

${ }^{43}$ F. Califano, N. Attico, F. Pegoraro, G. Bertin, and S. Bulanov, Phys. Rev. Lett. 86, 5293 (2001).

${ }^{44}$ D. Del Sarto, F. Califano, and F. Pegoraro, Phys. Rev. Lett. 91, 235001 (2003).

${ }^{45}$ K. Fujimoto, Geophys. Res. Lett. 41, 2721, doi:10.1002/2014GL059893 (2014).

${ }^{46}$ J. M. Urrutia and R. L. Stenzel, Phys. Plasmas 21, 122107 (2014).

${ }^{47}$ S. Y. Huang, H. S. Fu, Z. G. Yuan, A. Vaivads, Y. V. Khotyaintsev, A. Retino, M. Zhou, D. B. Graham, K. Fujimoto, F. Sahraoui, X. H. Deng, B. Ni, Y. Pang, S. Fu, D. D. Wang, and X. Zhou, J. Geophys. Res.: Space Phys. 121, 6639, doi:10.1002/2016JA022650 (2016).

${ }^{48}$ D. B. Graham, A. Vaivads, Y. V. Khotyaintsev, and M. Andr, J. Geophys. Res.: Space Phys. 121, 1934, doi:10.1002/2015JA021239 (2016).

${ }^{49}$ O. L. Contel, A. Retinó, H. Breuillard, L. Mirioni, P. Robert, A. Chasapis, B. Lavraud, T. Chust, L. Rezeau, F. D. Wilder, D. B. Graham, M. R. Argall, D. J. Gershman, P.-A. Lindqvist, Y. V. Khotyaintsev, G. Marklund, R. E. Ergun, K. A. Goodrich, J. L. Burch, R. B. Torbert, J. Needell, M. Chutter, D. Rau, I. Dors, C. T. Russell, W. Magnes, R. J. Strangeway, K. R. Bromund, H. K. Leinweber, F. Plaschke, D. Fischer, B. J. Anderson, G. Le, T. E. Moore, C. J. Pollock, B. L. Giles, J. C. Dorelli, L. Avanov, and Y. Saito, Geophys. Res. Lett. 43, 5943, doi:10.1002/ 2016GL068968 (2016).

${ }^{50}$ X. Tang, C. Cattell, J. Dombeck, L. Dai, L. B. Wilson, A. Breneman, and A. Hupach, Geophys. Res. Lett. 40, 2884, doi:10.1002/grl.50565 (2013).

${ }^{51}$ P. M. Bellan, Phys. Plasmas 20, 082113 (2013). 